\title{
Gender differences in sexual behaviors, sexual partnerships, and HIV among drug users in New York City
}

\author{
Judith Absalon • Crystal M. Fuller • \\ Danielle C. Ompad • Shannon Blaney • Beryl Koblin • \\ Sandro Galea · David Vlahov
}

Published online: 5 May 2006

(C) Springer Science+Business Media, Inc. 2006

\begin{abstract}
We compared sexual behaviors/partnerships and determined sexual risk correlates associated with HIV by gender among street-recruited drug users using chi-square tests and logistic regression. Men reported higher risk sexual behaviors, yet fewer high-risk sexual partners than women. After adjustment, HIV seropositive men were more likely than seronegatives to be older, MSM, use condoms, and have an HIV-infected partner. HIV seropositive women were more likely to be older, have an HIV-infected partner, and not use non-injected heroin. IDU was not associated with HIV. Prospective studies are needed to determine how genderspecific sexual behaviors/partnerships among drug users affect HIV acquisition.
\end{abstract}

Keywords Drug users · Sexual behaviors · Partnerships · HIV · Gender

J. Absalon · C. M. Fuller

Center for Infectious Disease Epidemiologic Research (CIDER), Department of Epidemiology, Mailman School of Public Health, Columbia University, New York, New York

C. M. Fuller · D. C. Ompad · S. Blaney · S. Galea · D. Vlahov Center for Urban Epidemiologic Studies (CUES), New York Academy of Medicine, New York, New York

B. Koblin

Laboratory of Infectious Disease Prevention, The New York Blood Center, New York, New York

J. Absalon $(\square)$

Department of Epidemiology, Mailman School of Public Health, Columbia University, Room 513, 722 West 168th Street,

New York, New York 10032

e-mail: ja234@columbia.edu

\section{Introduction}

Recent reports have indicated that HIV prevalence and incidence is decreasing among injection drug users (IDUs; Des Jarlais et al., 2000). HIV prevalence among IDUs in the late 1990's ranged from 2 to 10\% (Fuller et al., 2003) and incidence has been estimated at $0-1$ per 100 person-years in most published reports (Des Jarlais et al., 2000). Prevention efforts focused on reducing high-risk injection practices among IDUs have likely contributed to the decline of HIV among IDUs in the US (Semaan et al., 2002). Less dramatic declines have been noted among non-IDUs in whom HIV prevalence rates have been estimated up to $15 \%$ in published studies in the US and abroad (Chitwood, Comerford, and Sanchez, 2003). These high rates of HIV among injecting and non-injecting drug users suggest that high-risk sexual behaviors may be of increasing importance in HIV transmission in these populations.

Prior studies that have evaluated sexual risk behaviors in drug users have been largely among IDUs (Latkin, Mandell, and Vlahov, 1996). Of the reports that assessed sexual risk behaviors among non-IDUs, a clear association for sexually transmitted HIV has been demonstrated in crack users (Edlin et al., 1994). Few studies have been conducted on the sexual risk behaviors of IDUs in the context of the currently declining rates of HIV, during a period of decreased crack use (Department of Health and Human Services, 2003) or among a more diverse sample of non-IDUs such as those who use non-injection heroin, intranasal, or inhaled cocaine. In addition, while prior studies have identified gender-based differences in sexual behaviors in various populations, recent evidence among HIV-infected drug users have highlighted 
the need to further explore issues on the influence of sexual partner type including HIV serostatus of sexual partners (Semple, Patterson, and Grant, 2002).

To characterize the association of sexual behaviors and sexual partner type with HIV seropositivity among male and female drug users, we conducted a cross-sectional analysis of baseline data collected among IDUs and non-IDUs enrolled in two prospective cohort studies in New York City (NYC). The objectives of this analysis were to describe gender differences in sexual behaviors, and identify sexual behaviors and sexual partner characteristics associated with HIV seropositivity by gender.

\section{Methods}

Participants

Study participants were recruited using street outreach techniques into two ongoing studies from communities with high rates of illicit drug use. Research staff familiar with places in the community where drugs were bought or used, engaged potential participants in conversations about ongoing research conducted by the Center for Urban Epidemiologic Studies (CUES) at the New York Academy of Medicine. Those interested in participation were screened for eligibility at one of two community-based storefronts or the CUES research study van for enrollment into the HOPE cohort study, targeting non-IDUs, or the Hepatitis $\mathrm{C}$ cohort study, targeting newly initiated IDUs. HOPE study participants were eligible if they were between 15 and 40 years of age, reported noninjection use of heroin, crack or cocaine at least one time per week, but for no longer than 10 years, and had no history of injecting drugs. Eligibility for the Hepatitis $\mathrm{C}$ study included age 15-40 years and reported injection of heroin or cocaine at least once in the last 2 months but for no longer than 5 years.

\section{Measures}

Participants in each study were interviewed in a private room using a standardized drug and sexual risk survey. The survey included questions about sociodemographics, drug type and frequency of use, drug route of administration, drugusing companions, and sexual behaviors. Sociodemographic variables collected included: Age, race/ethnicity, education level, nationality, homelessness, and prior incarceration. We assessed whether participants used crack, cocaine, heroin, metamphetamines, hallucinogens, marijuana, or tranquiliz- ers and asked whether they injected, snorted, sniffed, or smoked each of these drugs. We collected information on sexual behaviors including age of sexual debut, drug use before, during, and after sexual acts, condom use, as well as gender and type of sexual partners. Sexual partner type was categorized as a steady, casual, or exchange-for-sex partner. A steady partner was defined as a person to whom participants felt strong emotional closeness; a casual partner was one with whom they were not emotionally close and an exchange-for-sex partner was a person with whom they traded sex for money or drugs. Participants were also interviewed about the HIV status and drug-using behaviors of each type of sexual partner.

Following each interview, participants received HIV pretest counseling and had blood drawn for serologic testing. Post-test counseling was provided 3 weeks later when participants returned for HIV results. Referrals for social and medical services were provided as appropriate during that time. HIV antibodies were detected using enzyme-linked immunosorbant assay (ELISA) for HIV types 1 and 2 (Abbott Laboratories, Abbott Park, IL) with a confirmatory Western Blot for HIV type 1 (Calypte Biomedical Corporation, Alameda, CA).

\section{Data analyses}

Baseline data from the Hepatitis $\mathrm{C}$ and HOPE studies were combined for this analysis and restricted to those who reported any oral, anal, or vaginal sex within the past 2 months. All analyses were stratified by gender. Sexual behavior and sexual partner variables were further evaluated by samegender sexual behavior. Men were defined as heterosexual, men who have sex only with men (exclusive MSM) or men who reported sex with men and women (MSM/W). Women were categorized as heterosexual, women who reported sex only with women (exclusive WSW) or women who report sex with men and women (WSW/M).

Gender stratified bivariate analyses were conducted to examine sociodemographic, drug use, sexual risk behavior, and sexual partner variables using chi-square or Fisher's exact tests for categorical variables and $t$-tests for continuous variables. Significant bivariate associations between exposure variables of interest and HIV seropositivity using $p$-values $<.10$ were used to help guide model building. Plausible interactions between gender and drug-using and sexual behavior variables were also explored using a $p$-value $<.20$ to help guide selection of variables for inclusion in the final model. Two separate final models using logistic regression were developed for men and women to determine the independent effect of each variable of interest as it related to HIV seropositivity. 


\section{Results}

Gender differences in sociodemographic and drug-use characteristics

One thousand and four participants were enrolled and interviewed between August 2000 and November 2003. Of these, 818 were sexually active within the prior 2 months and were included in this analysis. Table 1 provides details of participant demographic characteristics and drug-use type by gender. Men were younger (mean age 28 years versus 30 years, $p<.01$ ), more often Latino (63\% versus $41 \%$; $p<.01)$, and more likely to report recent homelessness $(61 \%$ versus $50 \% ; p<.01)$ or prior incarceration $(90 \%$ versus $79 \%$; $p<.01)$ compared to women. More men than women were IDUs $(29 \%$ versus $20 \% ; p<.01)$. Among non-IDUs, men were less likely to report crack use compared to women $(56 \%$ versus $55 \%, p<.01)$ and more likely than women to use marijuana $(77 \%$ versus $68 \%, p<.05)$ or hallucinogens $(20 \%$ versus $9 \%, p<.01)$. Intranasal cocaine and heroin use was similar between groups.

Gender differences in reported sexual behaviors and sexual partner characteristics

Reported sexual behaviors and characteristics of sexual partners within the preceding 2 months of study enrollment are detailed in Table 2 . The majority of participants were heterosexual; however, $11 \%$ of men and $20 \%$ of women reported same gendered sex. Five percent of men (50\% of MSM) and $13 \%$ of women ( $65 \%$ of WSW) reported sex with both men and women (data not shown). Approximately $29 \%$ of women reported a male partner who was an MSM (data not shown). Though most participants reported that they had a steady sexual partner, fewer men than women $(74 \%$ versus $88 \% ; p<.01)$ reported a steady partner. Men more often than women reported having a casual sexual partner $(52 \%$ versus $31 \% ; p<.01)$, multiple sexual partners $(54 \%$ versus $34 \% ; p<.01)$, and being high during sex $(84 \%$ versus $76 \%$; $p<.05)$. There were no gender differences with respect to having sex in exchange for money or drugs. Condom use overall was low for both men and women $(34 \%)$ and did not differ by gender or by type of sexual partner.

Among participants who reported having a steady sexual partner, men were less likely than women to have a drugusing steady partner ( $9 \%$ versus $23 \%, p<.01$, for IDU steady partner; $37 \%$ versus $70 \% p<.01$, for non-IDU steady partner). Among participants who reported an exchange-for-sex partner, men more frequently than women reported a nonIDU partner $(85 \%$ versus $67 \%, p<.01)$. Men and women reported similar rates of known HIV-infected sexual partners, irrespective of partner type.
HIV serostatus

Overall, HIV seropositivity was $9 \%$ and significantly lower among men than women $(7 \%$ versus $13 \%, p<.05$ ) (Table 2). This trend was similar when we compared HIV rates in heterosexual men $(5 \%)$ to those in heterosexual women $(12 \%, p<.01)$, and while MSM $(24 \%)$ had higher rates of HIV than WSW (17\%) the difference was not statistically significant. Further analysis of HIV rates by sexual orientation (data not shown) revealed that exclusive MSM had the highest rates of HIV (41\%) compared to MSM/W (9\%) or heterosexual men $(5 \%, p<.01$, Fisher's exact test). Rates of HIV among women were somewhat higher among WSW/M (20\%) compared to rates in exclusive WSW $(13 \%)$ or heterosexual women $(12 \%, p>.05$, Fisher's exact test).

Characteristics associated with HIV seropositivity

We explored demographic, sexual behavior, sexual partnership and drug use characteristics associated with HIV seropositive status stratified by gender on bivariate and multivariable analysis (Table 3). On bivariate analysis, HIV seropositive men were more likely to be older, of black race, and be better educated, while men who were recently homeless were less likely to be HIV seropositive than seronegatives. Sexual behavior and partner characteristics associated with HIV seropositivity in men included being an MSM, having an HIV-infected steady or casual/exchange-for-sex partner, having a non-IDU steady sexual partner, and using a condom with steady sexual partners at least $50 \%$ of the time. No association was found between HIV seropositivity and condom use with casual or exchange-for-sex partners in men.

After adjustment for age and recent homelessness, being an MSM ( $\mathrm{AOR}=5.59)$, having an HIV-infected steady ( $\mathrm{AOR}=7.22)$, or non-steady partner $(\mathrm{AOR}=15.36)$, and using condoms consistently with steady partners $(\mathrm{AOR}=3.65)$ were significantly associated with being HIV seropositive compared with being seronegative among men.

Among women, on bivariate analysis (Table 3), increasing age and black race were characteristics associated with being HIV seropositive. Women with multiple sexual partners, an HIV-infected steady, or non-steady partner and who reported consistent condom use with steady partners were more likely to be HIV seropositive than seronegative. Intranasal heroin use was associated with a lower likelihood of being HIV seropositive among non-IDU women compared to non-IDUs who did not use heroin.

After adjustment for age, HIV seropositive women were less likely to report intranasal heroin use $(\mathrm{AOR}=0.35)$ and more likely to have an HIV-infected steady sexual partner $(\mathrm{AOR}=9.39)$, or non-steady partner $(\mathrm{AOR}=6.41)$ 
Table 1 Comparison of demographic characteristics and drug types used in male versus female drug users, New York City 2000-2003

\begin{tabular}{|c|c|c|c|c|c|c|}
\hline & \multicolumn{2}{|c|}{ Men $(N=590)$} & \multicolumn{2}{|c|}{ Women $(N=228)$} & \multirow[b]{2}{*}{$d f^{a}$} & \multirow[b]{2}{*}{$\chi^{2 b}$} \\
\hline & $\bar{N}$ & $\%$ & $\bar{N}$ & $\%$ & & \\
\hline Mean age in years $(S D)$ & 28 & $(6.25)$ & 30 & $(6.61)$ & 816 & $-4.32^{c, *}$ \\
\hline \multicolumn{7}{|l|}{ Race/ethnicity } \\
\hline Latino & 371 & 63 & 91 & 41 & \multirow[t]{4}{*}{3} & \multirow[t]{4}{*}{$48.38^{*}$} \\
\hline Black $^{d}$ & 183 & 31 & 98 & 44 & & \\
\hline White & 15 & 3 & 26 & 12 & & \\
\hline Other & 21 & 7 & 9 & 4 & & \\
\hline \multicolumn{7}{|c|}{ Completed less than high school } \\
\hline Yes & 323 & 55 & 134 & 59 & \multirow[t]{2}{*}{2} & \multirow[t]{2}{*}{4.06} \\
\hline No & 265 & 45 & 84 & 41 & & \\
\hline \multicolumn{7}{|c|}{ Homeless in preceding 6 months } \\
\hline Yes & 357 & 61 & 115 & 50 & \multirow[t]{2}{*}{1} & \multirow[t]{2}{*}{$6.97^{*}$} \\
\hline No & 232 & 39 & 113 & 50 & & \\
\hline \multicolumn{7}{|l|}{ Ever arrested } \\
\hline Yes & 527 & 90 & 180 & 79 & \multirow[t]{2}{*}{1} & \multirow[t]{2}{*}{$15.63^{*}$} \\
\hline No & 62 & 10 & 48 & 21 & & \\
\hline \multicolumn{7}{|l|}{ Injection drug user (ever) } \\
\hline Yes & 173 & 29 & 46 & 20 & \multirow[t]{2}{*}{1} & \multirow[t]{2}{*}{$7.02^{*}$} \\
\hline No & 417 & 71 & 182 & 80 & & \\
\hline \multicolumn{7}{|c|}{ Non-injection drug user (last 6 months, $N=812$ ) } \\
\hline Crack & & & & & & \\
\hline Yes & 326 & 56 & 150 & 66 & \multirow[t]{2}{*}{1} & \multirow[t]{2}{*}{$7.23^{*}$} \\
\hline No & 259 & 44 & 77 & 34 & & \\
\hline \multicolumn{7}{|l|}{ Sniff/snorts cocaine } \\
\hline Yes & 407 & 69 & 146 & 64 & \multirow[t]{2}{*}{1} & \multirow[t]{2}{*}{2.08} \\
\hline No & 178 & 30 & 81 & 36 & & \\
\hline Smokes/snorts heroin & & & & & & \\
\hline Yes & 337 & 58 & 121 & 47 & 1 & 1.23 \\
\hline No & 248 & 42 & 106 & 53 & & \\
\hline Tranquilizers & & & & & & \\
\hline Yes & 149 & 26 & 160 & 71 & 1 & 1.37 \\
\hline No & 436 & 74 & 67 & 29 & & \\
\hline Marijuana & & & & & & \\
\hline Yes & 448 & 77 & 155 & 69 & 1 & $5.89^{* *}$ \\
\hline No & 137 & 23 & 72 & 31 & & \\
\hline Snort/smokes methamph & & & & & & \\
\hline Yes & 13 & 2 & 1 & 99 & - & $-^{e}$ \\
\hline No & 572 & 98 & 226 & 1 & & \\
\hline Hallucinogens & & & & & & \\
\hline Yes & 117 & 20 & 20 & 9 & 1 & $14.60^{*}$ \\
\hline No & 468 & 80 & 207 & 91 & & \\
\hline
\end{tabular}

${ }^{a}$ Degree of freedom.

${ }^{b} \chi^{2}$ : Chi-square statistic.

${ }^{c} t$-Statistic.

${ }^{d}$ Black race/ethnicity includes self-identified African American, black Caribbean, and African participants.

${ }^{e}$ Fisher's exact test was used, which does not have a formal test statistic.

${ }^{*} p<.01 .{ }^{* *} p<.05$.

compared with HIV seronegative women. Injection drug use was not associated with HIV seropositivity among men or women and was not further considered in the multivariate analysis.

\section{Discussion}

Among this sample of illicit drug users, we identified prominent gender differences with respect to sexual behaviors 
Table 2 Comparison of differences in sexual behaviors, sexual partner characteristics and HIV seropositivity of male vs. female drug users, New York City 2000-2003

Participant sexual behaviors ${ }^{a} N=818$

\begin{tabular}{|c|c|c|c|c|c|c|}
\hline \multicolumn{7}{|c|}{ Participant sexual behaviors $^{a} N=818$} \\
\hline & \multicolumn{2}{|c|}{$\operatorname{Men}(N=590)$} & \multicolumn{2}{|c|}{ Women $(N=228)$} & \multirow[b]{2}{*}{$d f^{b}$} & \multirow[b]{2}{*}{$\chi^{2 c}$} \\
\hline & $\bar{N}$ & $\%$ & $\bar{N}$ & $\%$ & & \\
\hline \multicolumn{7}{|l|}{ Sexuality } \\
\hline \multicolumn{7}{|l|}{ Heterosexual } \\
\hline Yes & 523 & 89 & 182 & 80 & 1 & $10.74^{*}$ \\
\hline \multicolumn{7}{|l|}{$\mathrm{MSM}$ or $\mathrm{WSW}^{d}$} \\
\hline Yes & 67 & 11 & 46 & 20 & & \\
\hline \multicolumn{7}{|c|}{ Currently lives with sexual partner } \\
\hline Yes & 211 & 36 & 135 & 59 & 1 & $37.04^{*}$ \\
\hline No & 379 & 64 & 93 & 41 & & \\
\hline \multicolumn{7}{|c|}{ Reports a steady sexual partner } \\
\hline Yes & 437 & 74 & 201 & 88 & 1 & $18.45^{*}$ \\
\hline No & 151 & 26 & 27 & 12 & & \\
\hline \multicolumn{7}{|c|}{ Reports a casual sexual partner } \\
\hline Yes & 304 & 52 & 70 & 31 & 1 & $28.19^{*}$ \\
\hline No & 280 & 48 & 154 & 69 & & \\
\hline \multicolumn{7}{|c|}{ Reports an exchange-for-sex partner } \\
\hline Yes & 158 & 27 & 71 & 31 & 1 & 1.55 \\
\hline No & 432 & 73 & 157 & 69 & & \\
\hline \multicolumn{7}{|l|}{ Multiple sexual partners ${ }^{e}$} \\
\hline Yes & 317 & 54 & 78 & 34 & 1 & $25.09^{*}$ \\
\hline No & 273 & 46 & 150 & 66 & & \\
\hline \multicolumn{7}{|l|}{ Often high during sex ${ }^{f}$} \\
\hline Yes & 487 & 84 & 173 & 76 & 1 & $0.01^{* *}$ \\
\hline No & 95 & 16 & 55 & 24 & & \\
\hline \multicolumn{7}{|c|}{ Condom use with sexual partners } \\
\hline 0 to $<25 \%$ of the time & 283 & 48 & 121 & 53 & 3 & 2.47 \\
\hline 25 to $<50 \%$ of the time & 36 & 6 & 15 & 7 & & \\
\hline 50 to $<75 \%$ of the time & 58 & 10 & 22 & 10 & & \\
\hline $75-100 \%$ of the time & 212 & 36 & 69 & 30 & & \\
\hline
\end{tabular}

Characteristics of sexual partners

\begin{tabular}{|c|c|c|c|c|c|c|}
\hline \multicolumn{7}{|c|}{ Steady sexual partners ${ }^{g}$} \\
\hline \multicolumn{7}{|c|}{ Steady partner was ever IDU ${ }^{h}$} \\
\hline Yes & 39 & 9 & 45 & 23 & \multirow[t]{2}{*}{1} & \multirow[t]{2}{*}{$22.90^{* *}$} \\
\hline No & 391 & 91 & 149 & 77 & & \\
\hline \multicolumn{7}{|c|}{ Steady partner is a non-IDU ${ }^{h}$} \\
\hline Yes & 157 & 37 & 138 & 70 & \multirow[t]{2}{*}{1} & \multirow[t]{2}{*}{$60.64^{* *}$} \\
\hline No & 272 & 63 & 59 & 30 & & \\
\hline \multicolumn{7}{|c|}{ HIV-infected steady sexual partner } \\
\hline Yes & 21 & 5 & 12 & 6 & \multirow[t]{2}{*}{1} & \multirow[t]{2}{*}{0.38} \\
\hline No & 416 & 95 & 189 & 94 & & \\
\hline \multicolumn{7}{|c|}{ Casual sex partners $^{i}$} \\
\hline \multicolumn{7}{|c|}{ Casual partner was ever IDU ${ }^{h}$} \\
\hline Yes & 33 & 12 & 3 & 5 & \multirow[t]{2}{*}{1} & \multirow[t]{2}{*}{2.61} \\
\hline No & 238 & 88 & 57 & 95 & & \\
\hline \multicolumn{7}{|c|}{ Casual sex partner is a non-IDU ${ }^{h}$} \\
\hline Yes & 162 & 56 & 37 & 58 & \multirow[t]{2}{*}{1} & \multirow[t]{2}{*}{0.18} \\
\hline No & 125 & 44 & 27 & 42 & & \\
\hline \multicolumn{7}{|c|}{ HIV-infected casual sexual partner ${ }^{j}$} \\
\hline Yes & 8 & 3 & 3 & 6 & \multirow[t]{2}{*}{-} & \multirow[t]{2}{*}{-} \\
\hline No & 242 & 97 & 50 & 94 & & \\
\hline
\end{tabular}


Table 2 Continued

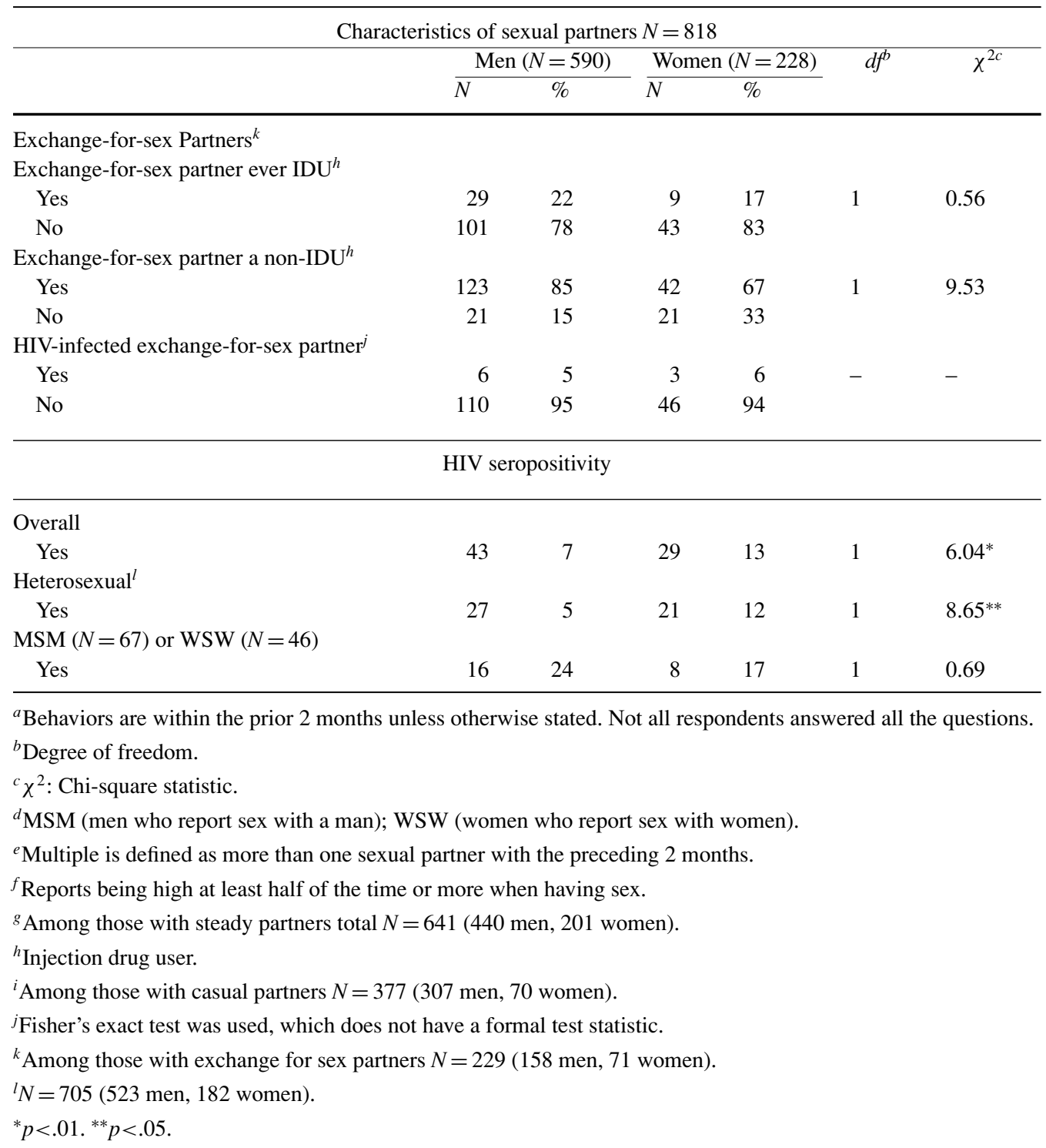

and sexual partner characteristics. HIV seroprevalence in this group was highest among drug-using MSM. Prevalence among heterosexual women was nearly double that of heterosexual men and rates among WSW were nearly four times that of heterosexual men. This significantly higher HIV prevalence in MSM and women compared to heterosexual men did not appear to be attributable to drug-using risk behaviors, but instead to high-risk sexual behaviors. In addition, sexual partner characteristics were primary factors associated with HIV for both men and women. A notable gender difference identified in this study was the difference in risk of steady compared to casual partnerships. Specifically, our data suggest that characteristics of steady partnerships contributed to the higher rates of HIV among women, while characteristics of non-steady partnerships contributed to rates among men. The differential influence of steady versus casual partners in men and women should be inter- preted with caution however and requires further verification in larger scaled studies.

Men engaged in more high-risk sexual behaviors when compared to women. Despite this, women had higher rates of HIV than heterosexual men. One possible explanation for these findings is that while men engaged in higher personal sexual risk behaviors, women more often reported sex with higher risk partners (IDU and non-IDU steady sexual partners). In addition, recent reports have suggested that the increasing rates of HIV infection among women may be in part related to sex with MSM who also have sex with women and that this group of men may be acting as a "bridge" between risk groups (Kalichman and Roffman, 1998).

HIV seropositivity rates in WSW were, however, slightly higher (17\% versus 11-12\%) than heterosexual women. A recent analysis conducted among this same study sample compared heterosexual women with WSW and found 
Table 3 Final adjusted models of factors associated with HIV seropositivity among male and female drug users, New York City 2000-2003

\begin{tabular}{|c|c|c|c|c|c|c|c|c|}
\hline Parameter & $\mathrm{OR}^{a}$ & $95 \% \mathrm{CI}^{b}$ & $d f^{c}$ & $\chi^{2 d}$ & $\mathrm{AOR}^{e}$ & $95 \% \mathrm{CI}^{b}$ & $\mathrm{~d} f^{c}$ & $\chi^{2 d}$ \\
\hline \multicolumn{9}{|c|}{ Men } \\
\hline Mean age $(S D)^{f}$ & 1.10 & $1.05,1.16$ & 1 & $14.04^{*}$ & 1.08 & $1.02,1.14$ & 1 & 6.44 \\
\hline Black Race $^{g}$ & 3.8 & $2.01,7.20$ & 1 & $16.78^{* *}$ & - & - & - & - \\
\hline Homeless in preceding 6 months & 0.32 & $0.16,0.64$ & 1 & $8.16^{*}$ & 0.29 & $0.14,0.62$ & 1 & $10.08^{* *}$ \\
\hline Education $^{h}$ & 3.00 & $1.31,6.87$ & 1 & $6.76^{* *}$ & - & - & - & - \\
\hline $\operatorname{MSM}^{i}$ & 5.22 & $2.65,10.27$ & 1 & $25.32^{*}$ & 5.59 & $2.50,12.49$ & 1 & $17.62^{*}$ \\
\hline HIV-infected steady sexual partner ${ }^{j}$ & 13.43 & $5.12,34.26$ & 1 & $14.92^{*}$ & 7.22 & $1.89,27.52$ & 1 & $8.39^{*}$ \\
\hline $\begin{array}{l}\text { HIV-infected casual or } \\
\quad \text { exchange-for-sex partner }{ }^{k}\end{array}$ & 21.20 & $5.58,80.46$ & 1 & $21.45^{*}$ & 15.36 & $3.06,77.06$ & 1 & $11.02^{*}$ \\
\hline $\begin{array}{l}\text { Condom use with steady sexual partner } \\
25-50 \% \text { of the time }\end{array}$ & 4.00 & $0.79,20.16$ & 1 & $2.79^{* * *}$ & 2.72 & $0.39,19.11$ & 1 & 1.01 \\
\hline $\begin{array}{l}\text { Condom use with steady sexual partner } \\
50-75 \% \text { of the time }\end{array}$ & 2.69 & $0.70,10.33$ & 1 & $2.19^{* * *}$ & 4.47 & $0.96,20.89$ & 1 & 3.62 \\
\hline $\begin{array}{l}\text { Condom use with steady sexual partner } \\
75-100 \% \text { of the time }\end{array}$ & 3.49 & $1.48,8.21$ & 1 & $6.82^{*}$ & 3.64 & $1.50,8.87$ & 1 & $8.13^{*}$ \\
\hline Steady sexual partner is a non-IDU ${ }^{m}$ & 1.84 & $0.97,3.49$ & 1 & $3.48^{* * *}$ & - & - & - & - \\
\hline Injection drug user & 0.55 & $0.25,1.22$ & 1 & 2.16 & - & - & - & - \\
\hline \multicolumn{9}{|c|}{ Women } \\
\hline Mean age $(S D)^{f}$ & 1.11 & $1.04,1.19$ & 1 & $8.77^{*}$ & 1.13 & $1.05,1.22$ & 1 & $10.07^{*}$ \\
\hline Black Race ${ }^{g}$ & 8.18 & $1.05,63.55$ & 1 & $4.03^{* *}$ & - & - & - & - \\
\hline Multiple sexual partners $^{n}$ & 2.72 & $1.23,6.00$ & 1 & $6.15^{* *}$ & - & - & - & - \\
\hline HIV-infected steady sexual partner ${ }^{j}$ & 8.08 & $3.43,40.79$ & 1 & $9.77^{*}$ & 9.39 & $2.36,37.37$ & 1 & $10.10^{*}$ \\
\hline $\begin{array}{l}\text { HIV-infected casual or } \\
\quad \text { exchange-for-sex partner }{ }^{k}\end{array}$ & 3.12 & $0.52,18.59$ & 1 & $4.76^{* *}$ & 6.41 & $1.11,37.06$ & 1 & $4.3^{* *}$ \\
\hline $\begin{array}{l}\text { Condom use with steady sexual partner } \\
25-50 \% \text { of the time }\end{array}$ & 5.17 & $0.86,31.19$ & 1 & $3.21^{* * *}$ & - & - & - & - \\
\hline $\begin{array}{l}\text { Condom use with steady sexual partner } \\
50-75 \% \text { of the time }\end{array}$ & 5.17 & $1.15,23.33$ & 1 & $4.56^{* *}$ & - & - & - & - \\
\hline $\begin{array}{l}\text { Condom use with steady sexual partner } \\
75-100 \% \text { of the time }{ }^{n}\end{array}$ & 2.03 & $0.86,4.79$ & 1 & $2.63^{* * *}$ & - & - & - & - \\
\hline $\begin{array}{l}\text { Smoked or snorted heroin in the past } \\
6 \text { months }\end{array}$ & 0.33 & $0.13,0.80$ & 1 & $4.42 *$ & 0.35 & $0.14,0.84$ & 1 & $5.49^{* *}$ \\
\hline Injection drug user & 0.42 & $0.12,1.44$ & 1 & 1.92 & - & - & - & - \\
\hline
\end{tabular}

${ }^{a}$ Odds ratio.

${ }^{b}$ Lower, upper confidence interval.

${ }^{c}$ Degree of freedom.

${ }^{d} \chi^{2}$ : Chi-square statistic.

${ }^{e}$ Adjusted odds ratio.

${ }^{f}$ Age is included as a continuous variable in years and $S D$ : standard deviation.

${ }^{g}$ Black race/ethnicity includes self-identified African American, black Caribbean, and African participants.

${ }^{h}$ Education; HS education or equivalent and higher compared to less than high school or equivalent.

${ }^{i}$ Men who have sex with men.

${ }^{j}$ Reports having only one partner (steady) who is HIV-infected.

${ }^{k}$ Partner with whom money or drugs were exchanged for sex in the preceding 6 months.

${ }^{l}$ Compared to participants who report condom use 0 to $<25 \%$ of the time with sex.

${ }^{m}$ Injection drug user.

${ }^{n}$ Multiple is defined as more than one sexual partner with the preceding 2 months.

${ }^{*} p<.01 .{ }^{* *} p<.05 .{ }^{* * *} p<.20$. 
that WSW were significantly more likely than heterosexual women to report sexual partnerships with MSM (Ompad, Fuller, Galea, Del Vecchio, and Vlahov, 2004a). We were unable to further explore the relationships between WSW, WSW/M, and MSM because of limited numbers of participants in these subgroups. The findings presented in this and prior studies, however, highlight important implications for HIV transmission in communities of color. Our findings suggest that WSW and WSW/M may be a "bridging" population between MSM and heterosexual men. WSW and WSW/M have become populations of growing interest, in light of their high rates of risky drug use and sexual behaviors. Further study of these vulnerable women is needed to understand their risk behaviors and to develop appropriate interventions.

MSM had the highest prevalence of HIV among this group despite similar degree of condom use, number and characteristics of sexual partners (i.e., multiple sexual partners, number of casual partners, and HIV-infected partners) as heterosexual men. While sexual intercourse with other men confers significantly higher HIV risk than sex with women, additional factors may have played a role in the significantly higher rates of HIV among this group of MSM. For example, factors such as lack of disclosure and concurrent sex with women have been recently cited as important mechanisms of HIV transmission in women, but there is little information regarding the effect on HIV rates among MSM. The majority of MSM in this group were black or Latino. Black and Latino MSM continue to be at high risk for HIV, however only a few studies have examined primarily crack, heroin- and cocaine-using MSM of color with regard to HIV risk. In some reports, black and Latino MSM have been shown to be more likely to have concurrent relationships with women, be less likely to self-identify as "gay" or homosexual, engage in higher risk sexual behaviors, and be less likely to disclose their MSM status compared to white MSM (Solorio, Swendeman, and Rotheram-Borus, 2003). However, recent studies among drug-using MSM have noted that HIV seropositive MSM engaged in lower risk sexual behaviors (Fuller et al., 2005). We had too few MSM to fully explore specific risk factors among this group however we did identify higher sexual risk partnerships associated with HIV and this issue merits further exploration among drug-using MSM.

In both men and women, having a HIV-infected sexual partner was independently associated with being HIV seropositive. This association was true for casual or steady partners. Follow-up data are needed to determine if this association is as a result of high-risk sexual behaviors with known HIV-infected partners and resultant HIV acquisition or if HIV-infected drug users purposely "seek out" sexual partners who are known to be HIV-infected. The association of higher levels of condom use and HIV in men is consistent with this latter hypothesis and possibly represents adoption of safer sexual behaviors in these men. It is important to note however that overall condom use was quite low for both men and women, which highlights the need for effective prevention strategies to promote condom use among this vulnerable population. Sexual risk reduction efforts among populations of drug users will also need to address the differing issues of sexual risk (and perceived risk) among drug users who are HIV-infected versus those who are uninfected. The high prevalence of risky sexual behaviors noted among HIV-infected populations in treatment and care settings have demonstrated the need for more intense secondary prevention efforts among this population (Absalon, Della Latta, Wu, and El-Sadr, 2005). These efforts are likely to be even more challenging among HIV-infected drug users who may not be receiving ongoing HIV treatment and care.

With regards to drug use, we found no association between injection drug-use status and HIV seropositivity for either men or women. An earlier analysis conducted among this study sample found that HIV seroprevalence was higher among non-IDUs and injection status was not a significant correlate of HIV (Ompad et al., 2004b). To our knowledge, this finding has not been reported in previous studies of illicit drug users. The lack of association between injection drug use and HIV is also consistent with decreasing incidence of HIV among IDUs in the US (Semaan et al., 2002). These findings suggest that prevention messages targeting IDUs to reduce high-risk injection practices have been effective and support the increasing importance of sexual risk of HIV among IDUs. We also found no association between crack use and HIV seropositivity among women or men. This differs from earlier studies, particularly among crackusing women who have previously shown high rates of HIV attributed to unsafe sexual behaviors such as trading sex for drugs or money (Edlin et al., 1994). However, having an HIVinfected exchange-for-sex or casual partner demonstrated a strong association with HIV seropositive status highlighting behavior as opposed to drug type as an important predictor and better measure of HIV infection.

Our study differs from other published reports in that we found no association between race and HIV seropositivity. This may be related to the small sample of white participants in this study, which may have resulted in insufficient power to detect an association between race and HIV serostatus. It should be noted that the cross-sectional design of the study limits our ability to establish temporality between risk behaviors and HIV. In addition, participant behaviors and characteristics of partners were self-reported and could not be verified and may have generated socially desirable responses. However, previous research has found self-reported behavior from drug users to be valid and reliable (Anthony et al., 1991). Finally, we enrolled participants primarily from two distinct neighborhoods in NYC and these findings may 
not be generalizable to illicit drug users in other geographic areas. This line of research should therefore be conducted in other areas of the US to ascertain whether similar trends exist, particularly in areas with historically high HIV prevalence.

This study contributes significantly to the existing body of science conducted among vulnerable and high-risk populations. Prior studies that examined gender differences among drug users have focused on differences in drug-using behaviors or sampled small or restricted populations of drug users. In addition, given previously reported associations between high-risk sexual network characteristics and HIV seropositive status among adult IDUs, similar quantitative social network research among remaining and newly identified risk groups including IDU and non-IDU MSM, MSM/W, and women of color are necessary.

This study demonstrates the importance of gender differences in sexual behavior and sexual partner characteristics among a sample of IDUs and non-IDUs. These findings are particularly relevant as the risk of HIV in the US appears to be shifting from primarily drug-using behaviors to sexual behaviors. Additional prospective studies are needed to determine the specific effect of these differences on HIV acquisition and transmission.

Acknowledgments The authors would like to thank the study participants and the New York Academy of Medicine storefront and mobile unit staff. This study was funded by National Institute on Drug Abuse: DA 13146 and DA 12801.

\section{References}

Absalon, J., Della Latta, P., Wu, F., and El-Sadr, W. (2005). Sexual behaviors and condom use of HIV-infected men and women of color attending a treatment and care clinic. Journal of the National Medical Association, 97(7), 25S-31S.

Anthony, J. C., Vlahov, D., Celentano, D., Menon, A. S., Margolick, J., Cohn, S., et al. (1991). Self-report interview data for a study of HIV-1 infection among intravenous drug users: Description of methods and preliminary evidence on validity. The Journal of Drug Issues, 21(4), 739-757.

Chitwood, D. D., Comerford, M., and Sanchez, J. (2003). Prevalence and risk factors for HIV among sniffers, short-term injectors, and long-term injectors of heroin. Journal of Psychoactive Drugs, 35(4), 445-453.
Des Jarlais, D. C., Marmor, M., Friedmann, P., Titus, S., Aviles, E., Deren, S., et al. (2000). HIV incidence among injection drug users in New York City, 1992-1997: Evidence for a declining epidemic. American Journal of Public Health, 90(3), 352-359.

Department of Health and Human Services. (2003). Drug abuse warning network, emergency department trends, final estimates 1995 2002. Retrieved 2005, from http://www.dawninfo.samhsa.gov

Edlin, B. R., Irwin, K. L., Faruque, S., McCoy, C. B., Word, C., Serrano, Y., et al. (1994). Intersecting epidemics-crack cocaine use and HIV infection among inner-city young adults. Multicenter Crack Cocaine and HIV Infection Study Team. New England Journal of Medicine, 331(21), 1422-1427.

Fuller, C. M., Absalon, J., Ompad, D. C., Nash, D., Koblin, B., Blaney, S., et al. (2005). A comparison of HIV seropositive and seronegative young adult heroin- and cocaine-using men who have sex with men in New York City, 2000-2003. Journal of Urban Health, Mar 82(Suppl 1), i51-i61.

Fuller, C. M., Vlahov, D., Latkin, C. A., Ompad, D. C., Celentano, D. D., and Strathdee, S. A. (2003). Social circumstances of initiation of injection drug use and early shooting gallery attendance: Implications for HIV intervention among adolescent and young adult injection drug users. Journal of Acquired Immune Deficiency Syndromes, 32(1), 86-93.

Kalichman, S., and Roffman, R. A. (1998). Risk for HIV infection among bisexual men seeking HIV prevention services and risks posed to their female partners. Health Psychology, 17(4), 320 327.

Latkin, C. A., Mandell, W., and Vlahov, D. (1996). The relationship between risk networks' patterns of crack cocaine and alcohol consumption and HIV-related sexual behaviors among adult injection drug users: A prospective study. Drug and Alcohol Dependence, 42(3), 175-181.

Ompad, D. C., Fuller, C. M., Galea, S., Del Vecchio, S., and Vlahov, D. (2004a). HIV risk behaviors among young female drug users who have sex with women in New York City. Oral Presentation. Paper presented at the XV International Conference on AIDS, Bangkok, Thailand.

Ompad, D. C., Fuller, C. M., Galea, S., Wu, Y., Nash, D., Benjamin, E., et al. (2004b). Sexual risk is associated with HIV among injection and non-injection drug users: Implications for intervention strategies. Paper presented at the XV International Conference on AIDS, Bangkok, Thailand.

Semaan, S., Des Jarlais, D. C., Sogolow, E., Johnson, W. D., Hedges, L. V., Ramirez, G., et al. (2002). A meta-analysis of the effect of HIV prevention interventions on the sex behaviors of drug users in the United States. Journal of Acquired Immune Deficiency Syndromes, 30 (Suppl 1), S73-S93.

Semple, S., Patterson, T., and Grant, I. (2002). Gender differences in the sexual practices of $\mathrm{HIV}^{+}$heterosexual men and women. AIDS and Behavior, 6(1), 45-54.

Solorio, R., Swendeman, D., and Rotheram-Borus, M. J. (2003). Risk among young gay and bisexual men living with HIV. AIDS Education and Prevention, 15(Suppl A), 80-89. 